\title{
The ventricular-subventricular zone: a source of oligodendrocytes in the adult brain
}

\author{
Oscar Gonzalez-Perez* \\ Laboratory of Neuroscience, School of Psychology, DES Ciencias de la Salud, University of Colima, Colima, Mexico \\ *Correspondence: osglez@ucol.mx
}

Edited and reviewed by:

Egidio D'Angelo, University of Pavia, Italy

Keywords: neural stem cells, ventricular-subventricular zone, oligodendrocyte, myelin, white matter, oligodendrocyte precursor cells, remyelination, demyelinating disease

Demyelinating diseases are characterized by an extensive loss of oligodendrocytes and myelin sheaths from axolemma, which commonly result in disability in young adults. To date, there is no effective treatment against these neurological disorders. In the adult brain, there are neural stem cells (NSCs) that reside within a niche denominated ventricular-subventricular zone (V-SVZ) in the lateral wall of the cerebral ventricles. These NSCs give rise to neurons and oligodendrocytes that help preserve cellular homeostasis (Kriegstein and Alvarez-Buylla, 2009). Growing evidence indicates that $\mathrm{V}-\mathrm{SVZ}$ progenitor cells may represent an endogenous source of oligodendrocytes that can be useful to treat demyelinating diseases.

The e-Book "The ventricular-subventricular zone as a source of oligodendrocytes in the adult brain" has collected the most recent evidence regarding the mechanisms that modulate the proliferation, migration, quiescence, cell-fate choices and survival of oligodendrocyte precursors generated in the V-SVZ. This e-Book begins with an excellent study performed by the members of the Nada Zecevic's laboratory. They analyzed the effects of Sonic hedgehog (Shh) signaling on proliferation and specification of human cortical Olig2+ progenitors in vitro. They demonstrated that Shh increased the number of oligodendrocyte progenitors (OPCs). However, inhibition of endogenous Shh did not reduce the density of Olig2+ cells, which suggest an additional Shhindependent mechanism for oligodendrocyte generation (Ortega et al., 2013).

In the adult V-SVZ, the activation of NMDA receptors (NMDAR) increases the oligodendrocyte differentiation. Using the reporter gene luciferase, Carlos Matute et al. (Chapter 2) found that the activation of NMDR stimulates PKC, which precedes the activation of NADPH oxidase (NOX) (Cavaliere et al., 2013). Hence, the authors propose that NOX2 is involved in the transduction of the signal from NMDAR through PKC activation. This work also suggests that signaling through the cascade NMDAR-PKC-NOX2 generates ROS that, in turn, activate the $\mathrm{PI} 3 / \mathrm{mTOR}$ pathway and drives oligodendrogenesis.

Tyrosine kinase (Trk) receptors play a key role in regulating the function of V-SVZ NSCs. Particularly, V-SVZ neural progenitor cells express the epidermal growth factor receptor (EGFR). EGF strongly stimulates proliferation, survival, migration and differentiation into the oligodendrocyte lineage (Gonzalez-Perez and Alvarez-Buylla, 2011). In the chapter 3, Galvez-Contreras and colleagues compiled the current data regarding the role of EGFR and ErbB family signaling on V-SVZ NSCs and explained the downstream cascades involved in oligodendrogenesis (GalvezContreras et al., 2013). The authors also proposed a hypothetical model to support the EGF-induced oligodendrogenesis, which involves homodimerization between ErbB1 and ErbB3, which probably activate the PI3K or the STAT pathways. Inconsequence AKT is activated and the expression of Olig- 2 is induced, which determines oligodendroglial specification.

Endocannabinoids have been involved in oligodendrogenesis. As reported by the Dr. Malva's group (chapter 4), hemopressin, a modulator of cannabinoid receptor-1, can increase the oligodendroglial differentiation in V-SVZ progenitor cells (Xapelli et al., 2014). Their results suggest that hemopressin may be of potential interest to treat demyelinating diseases.

Erythropoietin (EPO) promotes the V-SVZ-derived neurogenesis and oligodendrogenesis. Sawamoto and coworkers recently demonstrated that the EPO derivative asialo-EPO promotes the differentiation of V-SVZ-derived OPCs into myelin-forming mature oligodendrocytes in the injured white matter of neonatal mice without causing erythropoiesis (Kaneko et al., 2013). These findings and the therapeutic proposal are discussed in the chapter 5 of this book.

In the chapter 6, the group of Prof. Dietzel found that the addition of equiosmolar supplements of mannitol or $\mathrm{NaCl}$ associated with microglia cells can influence the proliferation of OPCs (Kleinsimlinghaus et al., 2013). Interestingly, a maximal yield of OPCs is obtained by combining a cell culture medium with osmolarity $>280 \mathrm{mOsm}$ and low density of microglia cells.

Aging progressively decline in the proliferative capacity of V-SVZ progenitor cells that subsequently affects the incorporation of new cells in the olfactory bulb. In the chapter 7, Quiñones-Hinojosa's group presents the effects of aging across the V-SVZ-OB system. They surprisingly found that neurogenesis decline with aging, but oligodendrogenesis in the rostral migratory stream is not compromised (Capilla-Gonzalez et al., 2013).

In the chapter 8, Ken Arai and colleagues (Maki et al., 2013) summarized the recent studies on extrinsic (extracellular matrix, cerebrospinal fluid, vasculature) and intrinsic (transcription factors or epigenetic modifiers) factors, which mediate oligodendrocyte generation from the V-SVZ progenitor cells. These factors appear to decrease by aging and compromise the efficiency of remyelination into the brain. In the chapter 9, significant questions and hypotheses regarding this topic are analyzed by Agathou et al. (2013). In the last chapter, Zhang et al. compiled evidence 
that indicates that a pathological condition as stroke can increase oligodendrogenesis in the white matter and V-SVZ during brain repair (Zhang et al., 2013), which support the notion that many inflammatory cytokines regulate the V-SVZ cells (Gonzalez-Perez et al., 2012).

In summary, growing evidence indicates that V-SVZ NSCs are good candidates to establish stem cell-based therapies in demyelinating disorders. Nevertheless, chemical mediators and signaling pathways involved in all these processes are to be completely elucidated. Understanding the mechanisms that regulate the V-SVZ progenitor cells may help develop therapeutic approaches to treat demyelinating diseases.

\section{ACKNOWLEDGMENTS}

This work was financed by grants from Consejo Nacional de Ciencia y Tecnologia (CONACyT; INFR2014-224359), The National Institute of Health and the National Institute of Neurological Disorders and Stroke (NIH/NINDS; R01NS070024), and Programa de Mejoramiento al Profesorado (PROMEP; 103.5/12/4857).

\section{REFERENCES}

Agathou, S., Karadottir, R. T., and Kazanis, I. (2013). Niche derived oligodendrocyte progenitors: a source of rejuvenation or complementation for local oligodendrogenesis? Front. Cell. Neurosci. 7:188. doi: 10.3389/fncel.2013.00188

Capilla-Gonzalez, V., Cebrian-Silla, A., Guerrero-Cazares, H., Garcia-Verdugo, J. M., and Quinones-Hinojosa, A. (2013). The generation of oligodendroglial cells is preserved in the rostral migratory stream during aging. Front. Cell. Neurosci. 7:147. doi: 10.3389/fncel.2013.00147

Cavaliere, F., Benito-Munoz, M., Panicker, M., and Matute, C. (2013). NMDA modulates oligodendrocyte differentiation of subventricular zone cells through PKC activation. Front. Cell. Neurosci. 7:261. doi: 10.3389/fncel.2013.00261

Galvez-Contreras, A. Y., Quinones-Hinojosa, A., and Gonzalez-Perez, O. (2013). The role of EGFR and ErbB family related proteins in the oligodendrocyte specification in germinal niches of the adult mammalian brain. Front. Cell. Neurosci. 7:258. doi: 10.3389/fncel.2013.00258

Gonzalez-Perez, O., and Alvarez-Buylla, A. (2011). Oligodendrogenesis in the subventricular zone and the role of epidermal growth factor. Brain Res. Rev. 67, 147-156. doi: 10.1016/j.brainresrev.2011.01.001
Gonzalez-Perez, O., Gutierrez-Fernandez, F., Lopez-Virgen, V., Collas-Aguilar, J., Quinones-Hinojosa, A., and Garcia-Verdugo, J. M. (2012). Immunological regulation of neurogenic niches in the adult brain. Neuroscience 226, 270-281. doi: 10.1016/j.neuroscience.2012.08.053

Kaneko, N., Kako, E., and Sawamoto, K. (2013). Enhancement of ventricularsubventricular zone-derived neurogenesis and oligodendrogenesis by erythropoietin and its derivatives. Front. Cell. Neurosci. 7:235. doi: 10.3389/fncel.2013. 00235

Kleinsimlinghaus, K., Marx, R., Serdar, M., Bendix, I., and Dietzel, I. D. (2013). Strategies for repair of white matter: influence of osmolarity and microglia on proliferation and apoptosis of oligodendrocyte precursor cells in different basal culture media. Front. Cell. Neurosci. 7:277. doi: 10.3389/fncel.2013. 00277

Kriegstein, A., and Alvarez-Buylla, A. (2009). The glial nature of embryonic and adult neural stem cells. Annu. Rev. Neurosci. 32, 149-184. doi: 10.1146/annurev. neuro.051508.135600

Maki, T., Liang, A. C., Miyamoto, N., Lo, E. H., and Arai, K. (2013). Mechanisms of oligodendrocyte regeneration from ventricular-subventricular zone-derived progenitor cells in white matter diseases. Front. Cell. Neurosci. 7:275. doi: 10.3389/fncel.2013.00275

Ortega, J. A., Radonjic, N. V., and Zecevic, N. (2013). Sonic hedgehog promotes generation and maintenance of human forebrain Olig2 progenitors. Front. Cell. Neurosci. 7:254. doi: 10.3389/fncel.2013.00254

Xapelli, S., Agasse, F., Grade, S., Bernardino, L., Ribeiro, F. F., Schitine, C. S., et al. (2014). Modulation of subventricular zone oligodendrogenesis: a role for hemopressin? Front. Cell. Neurosci. 8:59. doi: 10.3389/fncel.2014.00059

Zhang, R., Chopp, M., and Zhang, Z. G. (2013). Oligodendrogenesis after cerebral ischemia. Front. Cell. Neurosci. 7:201. doi: 10.3389/fncel.2013.00201

Conflict of Interest Statement: The author declares that the research was conducted in the absence of any commercial or financial relationships that could be construed as a potential conflict of interest.

Received: 23 April 2014; accepted: 30 April 2014; published online: 16 May 2014. Citation: Gonzalez-Perez O (2014) The ventricular-subventricular zone: a source of oligodendrocytes in the adult brain. Front. Cell. Neurosci. 8:137. doi: 10.3389/fncel. 2014.00137

This article was submitted to the journal Frontiers in Cellular Neuroscience. Copyright (C) 2014 Gonzalez-Perez. This is an open-access article distributed under the terms of the Creative Commons Attribution License (CC BY). The use, distribution or reproduction in other forums is permitted, provided the original author(s) or licensor are credited and that the original publication in this journal is cited, in accordance with accepted academic practice. No use, distribution or reproduction is permitted which does not comply with these terms. 